\title{
Quand l'action concertée devient source de savoirs : étude sur les apprentissages réalisés par des acteurs impliqués dans une expérience de développement local en organisation communautaire en milieu rural
}

\author{
Lucie Morin $^{\text {a }}$
}

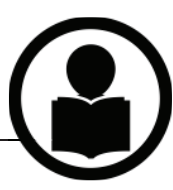

RÉSUMÉ. Depuis 1980, plusieurs municipalités rurales du Québec ont eu recours au développement local pour offrir de meilleures conditions de vie à leurs résidents. Les communautés rurales font face à des défis économiques, sociaux et environnementaux complexes exigeant que les personnes impliquées possèdent certaines connaissances et compétences. Cette recherche souhaite découvrir les phénomènes qui contribuent au développement de la capacité d'agir des personnes impliquées dans une démarche de développement local en milieu rural. Une étude de cas a été réalisée à partir de l'approche de développement local en organisation communautaire et de deux composantes du concept d'empowerment individuel : la participation et les compétences. Les résultats révèlent que la participation et l'acquisition de compétences dépendent de divers facteurs tels que les caractéristiques du profil des personnes, les facteurs favorisant la présence des personnes au sein du projet collectif, la disposition aux apprentissages ainsi que les modes d'apprentissage.

\begin{abstract}
Since 1980, several Quebec rural municipalities have turned to the local development approach to offer better living conditions to their residents. Rural communities face complex economic, social and environmental challenges requiring that the people involved have certain knowledge and skills. This research aims to discover the phenomena that contribute to the development of the ability to act for those involved in a local development process in rural areas. A case study was conducted from the local development approach in community organization and from two components of the concept of individual empowerment: participation and skills. The results reveal that the participation and the acquisition of knowledge depend on various factors such as the characteristics of the people's profile, the factors that foster the presence of people within the collective project, the readiness to learn and the learning methods.
\end{abstract}

\section{Introduction}

Au cours des dernières décennies, les transformations économiques et sociales provoquées par la mondialisation et la nouvelle économie du savoir exigent des restructurations et des adaptations plus significatives de la part des régions rurales que dans le reste de la société québécoise (Jean, 2003). L'écart continue de se creuser entre les pôles urbains et les secteurs ruraux au Québec, malgré les nombreuses possibilités associées aux milieux ruraux telles que la qualité de vie, les loisirs de plein air, l'accès à la propriété à moindre coût, etc.

En 2017, 25,8\% des Québécois habitaient en milieu rural (SRQ, 2018). On dénombre 1107 municipalités locales constituées selon les régimes municipaux généraux, dirigées par des élus locaux

${ }^{a}$ Doctorante en service social, Université de Montréal 
et agissant comme gouvernement de proximité auprès de la population (MAMH, 2020). Parmi elles, 711 désignent un territoire où résident moins de 2000 habitants (MAMH, 2020). Développé par l'Institut de la statistique du Québec (ISQ), l'indice de vitalité économique est composé de trois indicateurs qui sont le marché du travail, le niveau de vie et la dynamique démographique. En 2018, 233 entités locales avaient un indice de vitalité économique très faible (dernier quintile) et celles-ci se situent davantage dans les régions de la GaspésieÎles-de-la-Madeleine (33), de la Côte-Nord (19) et du Bas-Saint-Laurent (44) (Ladouceur, 2018).

On observe que de nombreux acteurs locaux sont mobilisés pour contrer la dévitalisation de leur milieu. Cette mise en action est propice à la création d'innovations sociales en matière de développement local (Alberio, 2015). Les initiatives qui ont eu des retombées positives sont celles où les acteurs du milieu ont procédé à l'identification des problématiques de développement qui leur sont propres, ont réalisé leur diagnostic territorial et ont rédigé une politique de développement territorial appropriée à leur territoire (Jean, 2015). L’approche de développement local peut relever plusieurs défis, mais cela nécessite, pour les acteurs impliqués, de posséder des expertises pointues et des savoirs techniques particuliers en lien avec des domaines bien précis. On peut supposer que ces expertises et ces savoirs sont, par moments, hors du champ de compétence des citoyens engagés dans de telles démarches, somme toute assez complexes.

L'ensemble de ces préoccupations a conduit au thème de cette recherche, qui se concentre sur l'acquisition de la capacité d'agir des acteurs qui font du développement local en milieu rural. Notre objectif général est de comprendre qui sont les personnes impliquées dans de telles démarches visant la revitalisation des communautés rurales et d'identifier ce qui favorise le développement de leurs connaissances et de leurs compétences. Cette recherche contribuera à la production de connaissances sur l'action concertée et, plus précisément, mettra en lumière les éléments qui favorisent le développement de la capacité d'agir des personnes engagées dans des démarches visant l'amélioration des conditions de vie de la population.

\section{Problématique}

Cette section de l'article vise à présenter l'évolution du développement local au Québec ainsi que le cadre conceptuel utilisé pour analyser l'objet d'étude.

\subsection{Développement local et ruralité}

Dans une vision suivant les travaux de l'économiste anglais Keynes sur l'intervention publique, le gouvernement fédéral adoptait l'Agricultural Rehabilitation and Development Act en 1961 afin d'identifier et de soutenir les régions présentant une diminution démographique et des difficultés économiques (Beaudet et Meloche, 2012). Au Québec, cette logique interventionniste de l'État s'est actualisée à travers le Plan de développement du Bureau d'aménagement de l'Est du Québec, organisme créé en 1963. Ainsi, l'État a décidé d'intervenir auprès de municipalités où les forces du marché libre ont généré des inégalités socioéconomiques (Jean, 2006). L'approche technocratique de planification qui influençait les interventions publiques, tant au palier fédéral que provincial, s'appuyait sur la théorie des pôles de croissance de Perroux (Joyal, 2015), qui relate que «le développement ne peut pas survenir partout, au même moment, avec la même intensité, mais repose plutôt sur la polarisation spatiale des activités » (Torre, 2015, p. 276) centralisées dans des endroits stratégiques à fort potentiel de développement économique. C'est dans les années 1970 que l'on constate les limites de la théorie des pôles de croissance. Non seulement les politiques publiques déployées n’ont pas été capables de produire les résultats attendus par rapport à l'homogénéisation économique et sociale de l'espace québécois (Ahtik, 1989), mais les clivages interrégionaux se sont accentués (Jean, 2012).

Puisque le modèle de croissance économique dominant ne contribuait pas à l'enrichissement de l'ensemble des collectivités du Québec ainsi qu'à l'épanouissement des individus, et que les personnes des milieux ruraux souhaitaient briser le cercle de la fatalité du destin, de nouveaux modèles de développement ont vu le jour. C'est au début des années 1980 que l'appellation développement local a commencé à être utilisée au Québec. Avant l'apparition de cette expression, on se référait à l'approche dite community-based economic development, 
utilisée aux États-Unis et au Canada anglais, et qu'on traduisait par le terme développement économique communautaire (Joyal, 2012). Selon Glon et Vachon (1996), le développement local «est une tentative d'appropriation ou de réappropriation des territoires, où un développement alternatif vient compléter les effets de la logique économique dominante, et où ce qui constitue traditionnellement des inégalités peut aussi être envisagé comme des différences » (p. 261).

Pour revitaliser une communauté, plusieurs citoyens doivent s'impliquer, mais la présence de certains d'entre eux est primordiale. Joyal, Pouquay et El-Batal (2005) mentionnent: «Il n'y a pas de développement local sans leader, ici, il est manifeste que l'élu exerce un rôle clé» (p. 210). Bon nombre d'élus sont heureux de s'impliquer dans des initiatives de développement local afin de favoriser le développement de leur communauté (Joyal et collab., 2005). Cependant, la motivation et la mobilisation des élus et des autres acteurs ne suffisent pas pour produire un développement viable et durable. Jean et Épenda Muteba Wa (2004) précisent qu'il est primordial que certains acteurs clés possèdent des savoirs techniques en matière de développement local tels que l'animation et la gestion de projets si des retombées positives veulent être observées.

Il est souhaitable qu'une pluralité d'acteurs puisse accompagner les citoyens dans une démarche de prise en charge collective. Or, plusieurs acteurs dénoncent le faible taux de participation de la population dans des démarches de développement local. Il est malheureux de constater que trop de citoyens n'ont pas conscience de leur capacité et du pouvoir d'agir qu'ils détiennent pour améliorer leurs conditions de vie et leur bien-être. Chaque personne possède des connaissances et des compétences qui peuvent être utiles pour soutenir la création et la mise en œuvre de projets collectifs. Joyal et ses collaborateurs (2005) relatent que l'augmentation de la participation citoyenne au sein d'initiatives locales exige un changement de mentalité ainsi que des activités de sensibilisation pour susciter la mobilisation des citoyens. Cette première étape s'inscrit bien dans une approche d'empowerment. Jean et Épenda Muteba Wa (2004) affirment que les communautés qui s'en sortent le mieux sont celles qui prennent des initiatives en se basant sur les compétences et les ressources locales.
D'où l'intérêt de réaliser une recherche afin de comprendre comment des citoyens engagés dans une démarche de développement ont augmenté leur capacité d'agir, et quelles sont les activités qui favorisent le développement de leurs connaissances et de leurs compétences. Ces nouvelles connaissances pourront guider les agents de développement dans leurs interventions afin de favoriser l'appropriation du pouvoir d'agir de l'ensemble des membres de leur communauté.

Parent (2011) souligne que plusieurs recherches qui s'intéressent au développement rural mettent davantage l'accent sur les dimensions économiques et politiques, et pas assez sur la dimension sociale afin de juger du potentiel de développement d'un milieu. Afin de pallier, de façon modeste, à ce manque de connaissances, notre recherche s'articule autour des questions suivantes :

1) Quels sont les phénomènes qui contribuent à la participation et au développement de compétences de personnes impliquées dans différents projets locaux en milieu rural?

2) Quels types d'activités favorisent la participation et la compréhension d'une démarche de développement local chez les personnes engagées dans le développement de leur communauté?

3) Quels sont les modes de fonctionnement qui favorisent la participation et le développement des compétences en matière de développement local?

\subsection{Cadre conceptuel}

Le développement local se définit à partir de deux grandes écoles de pensée qui ne mobilisent pas les mêmes stratégies. D'une part, il y a l'approche libérale qui, en s'appuyant presque exclusivement sur le secteur privé, en collaboration avec l'État, gratifie la croissance économique et la création d'emplois, sans tenir compte des besoins sociaux. D'autre part, l'approche progressive ou communautaire intègre les préoccupations sociales et économiques en préconisant l'entrepreneuriat individuel et collectif afin de développer des organisations sous différentes formes (Comeau, 2008; Fontan, 2003). C'est la seconde approche du développement local plus inclusive et sociale, aussi nommée approche socioéconomique par Bourque, Comeau, Favreau et Fréchette, (2007), qu'on retrouve dans la littérature des sciences sociales et que nous avons retenue comme composante de notre cadre conceptuel. 


\section{Le développement local en organisation communautaire}

L'approche du développement local en organisation communautaire caractérise fidèlement la démarche empruntée par l'initiative à l'étude. En effet, différents types d'acteurs (élus, citoyens, représentants d'organismes communautaires et de développement) ont décidé de prendre en charge leur développement afin de revitaliser leurs villages. Pour ce faire, ils ont procédé à plusieurs étapes, soit un état de situation, une analyse des besoins du milieu par le biais de consultations publiques, l'élaboration d'un projet de territoire et la mise en œuvre d'actions. Cette démarche repose sur les ressources locales (humaines, matérielles et financières) et sur la recherche de consensus, qui permet aux acteurs de se mobiliser vers des intérêts communs. Dans une perspective plus théorique, Bourque et Lachapelle (2010) définissent cette approche comme suit :

Le développement local des communautés vise l'identification par la population locale et les acteurs sociaux des problèmes et enjeux prioritaires des communautés et la réalisation d'initiatives visant à agir sur ces situations qui touchent généralement la dévitalisation socioéconomique et la détérioration des conditions de vie. (p. 35)

Ces auteurs précisent que cette stratégie permet d'améliorer et de consolider l'autonomie des acteurs par le développement d'initiatives collectives qui s'adressent à un territoire donné. Cette interprétation de l'approche du développement local en organisation communautaire met à l'avantplan les individus qui y collaborent. Étant donné la place centrale qu'ils occupent, il s'avère pertinent de comprendre quelle influence positive cette démarche a sur eux. Plus particulièrement, il importe de vérifier quels phénomènes issus de cette théorie interagissent avec le concept d'empowerment.

\section{L'empowerment}

Selon Ninacs (2008), le concept d'empowerment vise le développement de la capacité d'agir des personnes et des communautés soutenues en leur permettant de répondre elles-mêmes à leurs besoins :

L'empowerment repose sur la prémisse que les individus et les collectivités ont le droit de participer aux décisions qui les concernent et que les compétences requises par cette participation sont déjà présentes chez les individus et les collectivités ou que le potentiel pour les acquérir existe. (Ninacs, 2008, p. 15)

Cet auteur a élaboré un cadre conceptuel pour définir ce concept, qui est constitué de trois catégories, où chacune a ses propres caractéristiques : l'empowerment individuel, communautaire et organisationnel. Chaque catégorie est construite à partir de ses propres composantes. C'est la synergie qui se crée entre les trois catégories qui permet d'apprécier les changements souhaités ainsi que le développement de la capacité d'agir des personnes.

L'empowerment individuel est en lien direct à notre question de recherche. Ce type d'empowerment est constitué de quatre composantes : la participation, les compétences, l'estime de soi et la conscience critique (Ninacs, 2008). Sur le plan universitaire, la rédaction d'un mémoire présente certaines limites de faisabilité. Le temps et les ressources sont limités. Voilà pourquoi nous avons décidé de retenir seulement deux éléments sur quatre qui composent l'empowerment individuel, soit la participation et les compétences.

\section{La participation et les compétences}

De manière très sommaire, la participation se traduit par le fait qu'un individu, de par ses interrelations, contribue à des discussions, fait face à des choix et doit prendre des décisions (Ninacs, 2008). En ce qui a trait à la notion de compétences, Ninacs (2008) fait référence ici aux connaissances et aux habiletés qui permettent la participation et la réalisation d'actions dans le cadre d'une démarche collective.

Ces considérations théoriques sur le développement local en organisation communautaire et sur l'empowerment s'inscrivent en cohérence avec nos questions de recherche et la méthodologie retenue.

\section{Méthodologie}

Cette recherche qualitative a été réalisée dans le cadre d'une maitrise en service social sous la direction d'Yvan Comeau (Morin, 2016). Elle vise à approfondir la compréhension des phénomènes contributifs au développement de la capacité d'agir des personnes impliquées dans une démarche de développement local, à partir de leurs perceptions 
et de leur vécu. L'approche privilégiée est l'étude de cas (Stake, 2005). Nous avons anonymisé le cas afin de ne pas stigmatiser les municipalités concernées.

\subsection{Description du cas}

Le cas retenu est une démarche de développement local innovante qui engage quatre municipalités rurales dont les territoires totalisent une superficie de $434 \mathrm{~km}^{2}$. Elles furent colonisées vers la fin du xixe siècle sur la base de l'agroforesterie. Les municipalités ont été fondées entre 1858 et 1902. Elles ont toutes connu leur apogée démographique vers la fin des années 1950. Depuis ce temps, elles connaissent un déclin démographique. Selon l'Institut de la statistique du Québec (2020), entre 1951 et 2016, ces municipalités ont perdu respectivement $33 \%, 39 \%, 47 \%$ et $50 \%$ de leur population, une conséquence attribuée principalement au ralentissement des activités dans le domaine de l'agriculture et de la foresterie. Ces données affectent l'indice de vitalité économique tel qu'il a été calculé en 2016 par l'ISQ. Cette année-là, l'indice était négatif pour les quatre municipalités concernées $(-0,4214 ;-0,8039 ;-1,1494$ et $-5,5283)$, comparativement à un indice de 8,8436 pour leur municipalité régionale de comté (MRC).

Certaines données démographiques illustrent que la situation n'est pas sur le point de s'améliorer. Ces communautés démontrent un faible taux de natalité puisque, entre 2010 et 2015, dans chaque municipalité, il y a eu respectivement 17, 23, 32 et 66 naissances, pour un total de 138 . Ce nombre représente seulement $5,4 \%$ des naissances de l'ensemble du territoire de la MRC. Par ailleurs, le recensement du Canada de 2016 (Statistique Canada, 2021) dénombre 470 personnes ayant de 0 à 17 ans et 660 personnes de 65 ans et plus, des données qui témoignent d'une relève démographique déficitaire.

Malgré ces défis, les municipalités disposent d'atouts favorables à leur développement : un environnement unique caractérisé par des paysages pittoresques, une offre d'activités sportives et culturelles variée et un tissu associatif serré. Sur ce plan, un inventaire a répertorié 104 groupes ou associations dans des domaines variés d'activité : religieux, municipal, social, de santé et services sociaux, de développement local, éducatif, récréatif/sportif, culturel et environnemental.
Après trois années de réflexion et de consultation, un organisme à but non lucratif (OBNL) a été créé en mars 2013 pour orchestrer la mise en œuvre des priorités de développement identifiées collectivement. La mission dévolue à l'OBNL consiste à développer des projets durables et concertés au profit des quatre municipalités et de la qualité de vie de ses citoyens en mobilisant sa population, en créant des partenariats, en coordonnant la réalisation de projets et en mettant de l'avant des actions innovantes sur son territoire. Un second volet de la mission fait référence au transfert des savoir-faire à d'autres instances et sur d'autres territoires ainsi qu'à la mise en valeur de la culture rurale et de l'identité locale.

Notre recherche se concentre sur cette organisation spécifique qui intervient dans un territoire limité. Le conseil d'administration (CA) de l'OBNL est constitué de 13 personnes et se veut représentatif des quatre municipalités impliquées dans la démarche. Quatre catégories d'acteurs distincts le composent: a) un représentant de la MRC $(n=1), b)$ les maires $(n=4)$, c) des représentants d'organismes de développement local $(n=4)$ et d) des citoyens de chaque municipalité $(n=4)$. L'échantillon de référence regroupe 8 volontaires, soit 6 membres du CA (4 maires et 2 représentants d'organismes de développement local) ainsi que 2 professionnels responsables de soutenir et d'accompagner cette démarche. Une approche ciblée a permis de recruter les deux intervenants, car ils ont été identifiés par l'étudiante-chercheuse comme étant des acteurs significatifs en ce qui concerne le développement de la capacité d'agir des participants.

\subsection{Profil des participants}

L'échantillon est composé de quatre femmes et de quatre hommes. La moyenne d'âge du groupe est de 62 ans. Il est à noter que les six administrateurs sont mariés et que les deux professionnels sont considérés comme étant en union de fait. On peut qualifier la scolarité des participants comme étant très diversifiée. En effet, chez les administrateurs, on distingue les quatre profils suivants : personne sans diplôme d'études secondaires, diplômé d'études secondaires, diplômé d'études professionnelles et diplômé d'études universitaires. Les deux intervenants sélectionnés ont une scolarité différente : l'un a un diplôme d'études collégiales, alors que l'autre a un diplôme d'études universitaires. 
Enfin, le dernier élément qui définit le profil des administrateurs est l'implication du conjoint ou de la conjointe dans la communauté. Le tiers (2/6) des répondants affirme que leur conjoint(e) s'implique beaucoup dans leur milieu. Un autre tiers (2/6) mentionne que leur conjoint(e) s'implique peu. Le dernier tiers (2/6) déclare que leur conjoint(e) ne s'implique pas dans leur municipalité.

\subsection{Collecte de données}

La méthode de collecte de données privilégiée est celle de l'entretien semi-dirigé (Savoie-Zajc, 2010), effectué dans le cadre de rencontres individuelles. Deux guides d'entretien ont été élaborés : l'un pour les administrateurs et l'autre pour les accompagnateurs. Le guide destiné aux administrateurs était composé de 5 questions ouvertes et de 10 questions fermées permettant de dresser leur profil. En ce qui concerne le guide d'entretien conçu pour les intervenants, il comprenait 5 questions ouvertes et 5 énoncés dans la section réservée aux questions fermées. Les entretiens ont eu une durée moyenne de 76 minutes et elles ont toutes été enregistrées sous forme audio.

La collecte de données s'est échelonnée sur une période de 12 mois. Le premier entretien a eu lieu en février 2015 et le dernier, en janvier 2016. Un mode de fonctionnement a été convenu pour la réalisation des quatre premiers entretiens. Cette procédure comptait deux étapes: réalisation de l'entretien et production du verbatim. L'été 2015 a été le moment où des lectures préliminaires du matériel recueilli ont été faites. Un système de catégorisation des données a été élaboré pour certifier que les informations étaient en lien avec les phénomènes étudiés. Les quatre derniers entretiens ont été réalisés d'octobre 2015 à janvier 2016 en fonction d'une nouvelle procédure. Après chaque entretien, le verbatim et la codification des données ont été produits avant de planifier la prochaine rencontre avec un participant.

Le caractère inductif de la méthodologie qualitative a permis cette adaptation afin d'explorer en profondeur l'objet d'étude. Une analyse thématique du corpus de données a été réalisée (Sabourin, 2010) et sept fiches d'analyse ont été conçues autour des concepts théoriques centraux de la recherche : les composantes de l'empowerment, les acquis des participants et les modes d'apprentissage. Ce projet a reçu l'approbation des Comités d'éthique de la recherche de l'Université Laval.

\section{Résultats}

Les propos recueillis des participants par rapport à ce qui contribue au développement de leurs connaissances et de leurs compétences dans une démarche de développement local se divisent en quatre catégories : 1) les facteurs facilitants, 2) les obstacles, 3) la disposition des participants aux apprentissages en implication sociale et 4) les modes d'apprentissage. Reprenons chacun de ces éléments en détail.

\subsection{Facteurs facilitants}

Les entretiens ont révélé qu'il y a quatre facteurs facilitant la participation à une démarche de développement local et l'acquisition des savoirs : 1) la crédibilité/légitimité, 2) l'innovation et 3) les possibilités de reconnaissance personnelle.

\section{La crédibilité/ légitimité}

Tout d'abord, les participants ont décidé de s'impliquer dans le projet, car il le considérait comme étant crédible et légitime. La légitimité du projet repose sur deux éléments, selon les participants. Tout d'abord, il s'agit de la reconnaissance que les élus municipaux lui témoignent, étant donné que les maires y participent tous et que les quatre conseils municipaux ont adopté une résolution indiquant qu'ils soutenaient cette démarche. La seconde source d'influence provient des organisations provinciales à l'extérieur du territoire où se déroule l'initiative. Pour un participant, le projet est crédible quand des personnes ou des organisations qui n'ont aucun lien direct avec le projet lui reconnaissent du potentiel. Dans le cas présent, il s'agit de Solidarité rurale du Québec (SRQ) et de la Fédération québécoise des municipalités (FQM). Quand les participants constatent qu'un projet est source de fierté et reconnu par des élus ainsi que des partenaires influents, cela les incite à y participer.

\section{L'innovation}

Le caractère innovant du projet collectif constitue le deuxième facteur facilitant la participation des acteurs. La majorité des participants sont engagés dans le développement de leur communauté depuis plusieurs années et ils constatent malheureusement que les approches habituelles ne donnent pas les résultats escomptés. Cette prise de conscience effectuée par plusieurs participants les a prédisposés à s'ouvrir à de nouvelles manières de penser et d'agir. Concrètement, 
voici comment un participant le verbalise: "Tout seul, on ne sera pas capable d'arriver à combler le besoin à l'échelle des citoyens et à l'échelle de la municipalité. Il faut réellement regarder ensemble ce qu'on est capables de faire» (P6-18). L'urgence de la situation provoque une ouverture d'esprit et le goût de prendre des risques. En outre, le caractère inédit de l'innovation stimule l'intérêt d'autres leaders à expérimenter et à créer quelque chose de nouveau: "Qu'est-ce que ça va être? Quelle tournure ça va prendre? Qu'est-ce que ça peut donner?» (P4-14).

\section{Les possibilités de reconnaissance personnelle}

Les possibilités de reconnaissance représentent également un facteur qui influence la présence des personnes au sein du projet. La reconnaissance prend forme autour des sentiments de solidarité et de fierté qui sont vécus par les participants. La valeur de solidarité a été identifiée à travers les liens qui se sont développés entre les quatre municipalités, particulièrement entre les quatre élus, tout comme la cohésion et l'entraide qui caractérisent la dynamique du groupe. Cette cohésion entre les participants n'annihile pas les différences et les intérêts propres à chaque communauté. Au contraire, elle permet la collaboration en vue d'atteindre leur but commun. Les participants évoquent également la fierté qu'ils ressentent et qui émane des petits et grands succès qu'ils vivent ensemble. Ce sentiment de fierté influence positivement la présence des personnes au sein du projet. Les participants réalisent qu'aux plan individuels et collectifs, ils disposent de nombreux atouts pour revitaliser leur territoire.

\subsection{Obstacles}

Si plusieurs facteurs du contexte encouragent la présence de personnes dans une démarche de développement local en milieu rural, il existe à l'opposé plusieurs obstacles qui entravent la possibilité de réaliser des apprentissages. Les résultats de la recherche ont permis d'en identifier deux.

\section{Le manque de mutualité au sein du groupe}

Le premier élément qui indispose aux apprentissages concerne le groupe lui-même. En effet, le manque de mutualité dans le groupe - perçu ou réel
- peut nuire aux apprentissages. Les participants font référence à des expériences associées à des émotions désagréables et qui se sont transformées en frein à leur participation, notamment l'accueil des nouveaux membres, le processus décisionnel et les règles de fonctionnement du groupe.

\section{La perception négative et l'incrédulité des citoyens}

D'après les entretiens, un second obstacle est la perception négative et l'incrédulité des citoyens ou des personnes qui ne font pas partie prenante du projet, ce qui ébranle la motivation des personnes impliquées à poursuivre leur engagement. Les commentaires tels que « Ça ne marchera pas. Ça ne donnera rien» (P4-185) ont un effet néfaste sur le sentiment de contribuer à une œuvre commune. Une répondante explique les attitudes négatives des personnes externes au projet par de la résistance au changement et à l'innovation.

\subsection{Disposition des participants aux apprentissages en implication sociale}

L'analyse des verbatims des participants a permis de distinguer deux ensembles d'éléments concernant la disposition des personnes à effectuer des apprentissages : d'une part, leurs expériences et leurs connaissances en implication sociale et, d'autre part, les attitudes qui les ouvriront à de nouveaux apprentissages. Voyons comment cellesci sont décrites par les participants.

\section{Expériences et connaissances en implication sociale}

Les personnes engagées dans l'initiative à l'étude possédaient déjà un bagage de savoirs sur l'implication sociale avant de se joindre au projet collectif. En effet, elles avaient une longue expérience d'engagement dans leur milieu, étaient au fait de la politique municipale et connaissaient bien leur communauté ainsi que les enjeux de développement qui la caractérisent (évolution démographique, activité économique, rétention et attraction des populations, etc.). Comme le démontre le tableau qui suit, les participants consacrent de nombreuses heures par mois dans différents projets et cela fait partie de leur quotidien depuis de nombreuses années. 


\begin{tabular}{|c|c|c|c|c|}
\hline Participant & $\begin{array}{c}\text { Nbre d'heures } \\
\text { consacrées, par } \\
\text { mois, pour le } \\
\text { projet à l'étude }\end{array}$ & $\begin{array}{c}\text { Nbre d'heures con- } \\
\text { sacrées, par mois, } \\
\text { à participer à diffé- } \\
\text { rents projets de } \\
\text { leur communauté }\end{array}$ & $\begin{array}{c}\text { Nbre d'années } \\
\text { à occuper une } \\
\text { fonction } \\
\text { d'élus muni- } \\
\text { cipaux }\end{array}$ & $\begin{array}{c}\text { N'bre d'années } \\
\text { d'implication } \\
\text { dans leur com- } \\
\text { munauté }\end{array}$ \\
\hline \hline 2 & 8 à 10 & 35 & 10 & Inconnu \\
\hline 3 & 7 & 10 & 23 & 19 \\
\hline 4 & 3,5 & 11 & 4 & 7 \\
\hline 5 & 6 & 54 & 20 & 39 \\
\hline 6 & 5 à 6 & 20 & 18 & 23 \\
\hline 7 & 5 à 10 & 80 & 6 & 35 \\
\hline
\end{tabular}

Tableau 1 - Estimation par les participants du temps d'implication dans leur communauté au moment de l'entretien

Il est important de mentionner que cinq répondants sur six étaient des élus municipaux au moment où les entretiens ont eu lieu. La personne qui n'était pas élue au moment de l'entretien l'avait déjà été pendant de nombreuses années.

\section{Attitudes d'ouverture à de nowveaux apprentissages}

Les résultats de la recherche ont permis de distinguer trois attitudes qui peuvent faciliter les apprentissages chez les personnes impliquées dans une démarche de développement local.

Tout d'abord, l'attitude de bienveillance ou d'intérêt pour sa localité est manifeste chez quatre des six répondants et se trouve validée par le nombre d'années d'implication dans leur milieu, et ce, de différentes manières.

Ensuite, l'ouverture au changement individuel (ce qu'ils ont instauré et réalisé) et collectif (intérêt pour de nouvelles idées) représente une autre attitude susceptible de favoriser de nouveaux apprentissages en matière d'action concertée. En effet, deux répondantes associent leur présence dans la démarche collective à leur souhait de faire des découvertes, étant donné que le défi exige de s'ouvrir à de nouvelles perspectives et manières de penser et d'agir.

Enfin, la troisième attitude fait référence à la capacité de travailler de façon collaborative avec les autres. La culture de collaboration au sein du groupe est mise en rapport avec le fait de prendre le temps d'écouter les autres, de respecter leurs points de vue, de favoriser le partage de connaissances, d'informations et d'idées, etc. L'homogénéité du comité de communication s'est révélée être un environnement favorable aux apprentissages pour les femmes qui y participaient, étant donné qu'un climat de confiance y régnait, ce qui favorisait la prise de parole de chaque membre.
D'autres participants font plutôt allusion au rayonnement de l'équipe et à la mobilisation de différents réseaux pour soutenir leurs apprentissages. Le terme « réseau » désigne les acteurs interne (p. ex., employés d'une municipalité) et externe (p. ex., universités, FQM, MAMROT) du territoire visé par l'action qui peuvent être mobilisés afin d'obtenir les ressources nécessaires à la réalisation des actions.

\subsection{Modes d'apprentissage}

Trois modes d'apprentissage ont été révélés lors des entretiens : 1) les activités éducatives formelles, 2) les activités planifiées ayant conduit à des apprentissages non intentionnels et 3) celles favorisant des apprentissages non intentionnels informels.

\section{Les activités éducatives formelles}

Premièrement, il est possible de développer des connaissances et des compétences en participant à des activités formelles d'apprentissage qui se déroulent dans le cadre de cours ou d'activités de formation délibérément construites. À cet égard, trois participants sur six mentionnent avoir augmenté leur capacité d'agir sur différents thèmes (leadership, entrepreneuriat, utilisation de Facebook, etc.) en assistant à des formations. En plus d'acquérir des connaissances, trois membres affirment que les activités formelles permettent de penser autrement et de connaitre de nouvelles personnes.

\section{Les activités planifiées ayant conduit à des apprentissages non intentionnels}

Deuxièmement, les participants mentionnent pratiquer certaines activités leur permettant de faire des apprentissages non intentionnels. Quatre répondants (P4, P5, P6 et P7) font référence à la lecture 
(p. ex., les journaux) comme étant un excellent moyen de se tenir informé et de faire des apprentissages. De plus, trois participants (P2, P4 et P7) précisent qu'ils utilisent Internet pour effectuer des recherches afin de s'informer sur des sujets précis ou de suivre l'actualité en lien avec certains secteurs d'activité.

Dans une perspective plus relationnelle, deux participants mentionnent qu'ils privilégient les situations de groupe et les interactions avec d'autres personnes afin de soutenir l'acquisition de connaissances. À ce propos, trois activités semblent avoir été plus significatives pour les participants. Il s'agit d'une visite organisée en Gaspésie; de l'activité « Première impression", planifiée par les intervenants et qui avait comme objectif de permettre à l'ensemble des membres de connaitre le territoire sur lequel se déroule le projet collectif (quatre municipalités); et d'une mission d'une semaine qui s'est déroulée en France au printemps 2010. Cette mission a été inspirante pour plusieurs membres, car, en plus de leur permettre de découvrir un nouveau modèle de développement, ce voyage leur a permis de rencontrer des personnes qui, tout comme eux, se battent pour revitaliser leur communauté rurale. Cette activité a également eu des impacts positifs sur la cohésion du groupe. Le voyage a permis aux personnes d'apprendre à se connaitre davantage sur le plan personnel et des affinités se sont développées entre les membres (P8-109).

\section{Les activités favorisant des apprentissages non intentionnels informels}

Finalement, la recherche a mis en lumière que des apprentissages sont faits sans que cela ait été forcément planifié par quiconque. Il s'agit d'apprentissages non intentionnels informels induits par la participation à des activités collectives. Ainsi, trois membres nomment des apprentissages (connaissance de leur territoire et de leurs atouts) qu'ils associent à leur expérience d'implication communautaire, qui est considérable, faut-il le rappeler.

Des apprentissages ont également été faits en matière de mobilisation et de participation citoyennes. Sans avoir nécessairement eu des formations à ce sujet, deux répondants comprennent que ces principes sont au cœur du développement local en analysant rétrospectivement les succès et les échecs des projets auxquels ils ont collaboré.
Aussi, la création et le fonctionnement de l'OBNL supposent que les membres du CA se rencontrent sur une base mensuelle. Pour la participante 4, ces réunions sont considérées comme étant importantes pour soutenir la démarche de développement local qui est entamée (P4-137). Or, c'est aussi un lieu où elle fait de nombreux apprentissages, car différents sujets y sont traités et analysés.

\section{Interprétation}

Au-delà du processus par lequel se déroule une démarche en développement local, le succès de cette dernière repose sur les humains qui en sont les initiateurs et les principaux responsables. Les quatre prochaines sous-sections ont pour objectif de présenter des éléments de réponse à nos questions de recherche en tissant des liens entre les données empiriques et la littérature scientifique.

\subsection{Incitatifs à la présence des personnes au sein de projets collectifs et à l'acquisition de savoirs}

Les participants font référence à cinq facteurs assurant leur engagement au sein du projet étudié : 1) la légitimité, 2) la reconnaissance, 3) l'innovation, 4) le mode de fonctionnement et 5) le climat du groupe. C'est à travers cette participation que le développement des compétences peut s'opérer.

\section{La légitimité}

Les données démontrent que la légitimité accordée à la démarche est attribuable à la présence des maires, qui sont des leaders dans leur milieu. Vachon (1993) explique ce phénomène en mentionnant que les leaders sont indispensables dans une démarche de développement local, car les citoyens leur reconnaissent le droit et la compétence pour mener ce type de démarche collective. Les élus ont l'autorisation morale et légale d'instaurer des actions collectives puisqu'ils occupent une fonction officielle au sein d'une organisation légale - une municipalité - qui leur attribue ce type de pouvoir. En plus d'avoir le titre officiel d'élu, ces participants ont acquis diverses connaissances et compétences au cours de leurs nombreuses années d'implication aux échelles locale et territoriale. Pour toutes ces raisons, les maires sont des acteurs incontournables en développement local, autant 
pour les compétences et les ressources qu'ils peuvent fournir au groupe que pour la crédibilité qu'ils peuvent procurer au projet (Lachapelle et Bourque, 2020). Cela explique que l'implication des maires dans un projet collectif est un facteur qui contribue à l'augmentation de la capacité d'agir sur les plans individuel et collectif.

\section{La reconnaissance}

La reconnaissance accordée par des partenaires externes au projet est le second incitatif identifié par les participants comme ayant des impacts positifs sur leur présence au sein du projet. Lavoie et Panet-Raymond (2020) expliquent ce phénomène par le fait que l'humain a un besoin fondamental de reconnaissance pour donner un sens à ces gestes, et cette aspiration est comblée par ce type d'action de reconnaissance. Toutefois, d'une manière moins visible, ce type de reconnaissance augmente la capacité d'agir des acteurs. Comme l'explique Vachon (1993), le développement local n'est pas synonyme de «localisme». Le succès de toute initiative de développement repose sur la capacité des acteurs d'aller chercher du soutien et les ressources nécessaires pour permettre le développement de leur projet. On peut présumer que l'appui donné par les deux organisations provinciales facilite le développement d'alliances avec d'autres organisations locales, régionales ou provinciales afin d'avoir recours à des ressources et compétences diverses.

\section{L'innovation}

Le caractère innovant du projet collectif est apparu comme une dimension favorable autant pour assurer la présence des participants que pour nourrir leur désir d'acquérir des savoirs. Cela confirme les propos de Côté (2008), selon qui l'innovation est également une condition de maintien de la mobilisation en matière d'action collective. L'analyse des propos des participants permet de comprendre que l'innovation devient une option envisageable seulement lorsqu'ils font le constat que tout a été tenté pour revitaliser le milieu sans produire les résultats attendus. Ce désir de collaboration comporte des défis, car, comme le mentionne Alberio (2015) «l'innovation sociale n'est pas quelque chose d'évident à contrôler et à reproduire d'un territoire à l'autre. Elle est en fait encastrée dans un contexte socioterritorial, économique et politique spécifique» (p. 58). Cela illustre les difficultés qui se dressent devant les participants en matière d'apprentissages afin de s'approprier ce qui a été fait ailleurs et d'avoir la capacité de l'adapter en fonction des besoins et des ressources de leur territoire. Cela a été pour eux une occasion de faire des apprentissages et de contribuer au développement de leur capacité d'agir.

Une recherche menée par Simard et Leclerc (2008) explique que la société actuelle présente de nouveaux besoins qui exigent que les solutions débordent du cadre de la municipalité dite traditionnelle. Les participants expriment clairement leur volonté de contribuer au bien commun et cela est propice à l'innovation dans la façon de répondre aux besoins de la population. Ce constat corrobore les propos de Rivard (2000), selon qui les adultes sont motivés à faire des apprentissages quand les connaissances acquises leur permettent de trouver des solutions à un problème auquel ils font face. L'acquisition de savoirs chez les participants a été possible, car, après seulement quelques rencontres, leur présence au projet a été facilitée par l'émergence de solidarité et d'un sentiment de fierté qui a uni les membres du groupe. Les témoignages démontrent que chaque participant contribue activement à la réussite du projet collectif en partageant ses ressources : temps, connaissances, habiletés et réseau de contacts. Cette contribution est influencée positivement par le sentiment d'appartenance au groupe et à leur communauté (Duperré, 2004). Leur présence à chaque rencontre devient une occasion pour augmenter leur capacité d'agir, soit en renforçant certaines habiletés (prise de parole en public, analyse politique, etc.), soit en apprenant des connaissances en côtoyant d'autres personnes. Une participation soutenue et répétitive est favorable à l'acquisition d'apprentissages.

\section{Le mode de fonctionnement}

Les informations recueillies révèlent qu'il existe des facteurs qui font obstacle aux apprentissages et à la participation des personnes. Il s'agit du mode de fonctionnement et du climat qui règne au sein du groupe. Coureau (1993, cité dans Rivard, 2000) appuie ces résultats en affirmant qu'un adulte apprend s'il se sent intégré dans un groupe et si les membres peuvent avoir de nombreuses interactions. À ce sujet, Lavoie et Panet-Raymond (2020) affirment qu'il est capital 
que le mode de fonctionnement du projet soit harmonisé en fonction des intérêts et de la disponibilité des membres, si l'on souhaite que ces derniers s'engagent de manière assidue.

Les résultats démontrent que les professionnels ont pris en considération la disponibilité des participants dans la planification et dans la mise en ouvre du projet afin de respecter leurs limites et leur emploi du temps. En ce qui concerne les intérêts des participants, les résultats de l'étude relatent qu'ils possédaient des connaissances dans différents domaines, dont l'éducation, la politique et l'économie. La structure de l'OBNL leur offre l'occasion de s'impliquer dans différents comités en fonction de leurs aspirations. Le fait de pouvoir choisir le lieu où ils désirent s'impliquer a un effet direct sur l'augmentation de leur capacité d'agir. En effet, Rivard (2000) déclare qu'il faut prendre en considération l'expérience de l'apprenant afin de l'aider à faire des liens entre ce qu'il apprend et ce qu'il sait déjà.

\section{Le climat du groupe}

Les participants ont abordé l'incitatif du climat du groupe en faisant référence au sentiment de fierté qui les unit et à la valeur de la solidarité partagée par l'ensemble des membres. Selon Richard (2000), "le climat du groupe est très relié au type de communication qui y prévaut: le fait de se sentir en communication avec tous les membres semble un effet du climat ou de la vie du groupe» (p. 39).

C'est pourquoi Lavoie et Panet-Raymond (2020) suggèrent d'accorder une attention particulière à la qualité de la communication. Si cette dernière présente certaines lacunes, des conflits peuvent se développer et nuire à la mobilisation des personnes et, d'un même coup, empêcher l'acquisition d'apprentissages. Il faut alors privilégier les processus de collaboration, qui permettent aux membres du groupe d'échanger davantage entre eux afin de provoquer des occasions pour apprendre les uns des autres puisque la communication est un concept plus complexe que le simple acte de transmission d'un message (Richard, 2000). Hardina (2013) mentionne qu'une fréquence élevée d'interactions entre les membres d'une organisation permet l'établissement d'une relation de confiance. Le climat du groupe peut contribuer à l'épanouissement d'une personne ou, à l'opposé, la paralyser.
Une recherche de Duperré (2008) confirme que les émotions font partie intégrante des dynamiques internes des groupes mobilisés. Les émotions réciproques d'amitié et d'affection entre les membres sont très importantes, étant donné qu'elles contribuent à la cohésion du groupe. Les sentiments de solidarité et de fierté rapportés par les participants sont ancrés dans des émotions d'affection et de respect. Les résultats témoignent que les membres sont engagés autour d'un projet commun qui vise la revitalisation de leur milieu et où le plaisir est un élément qui les incite à travailler ensemble. Le projet devient alors un prétexte pour échanger et perfectionner ses connaissances sur un sujet donné, et ce, dans un contexte convivial.

Les résultats appuient les propos de Bonneau et Dupéré (2020) à l'effet qu'il existe des conditions qui favorisent la participation sociale des personnes au sein d'un projet collectif en milieu rural. Il est important de les prendre en considération, car la participation à des démarches collectives est un des moyens contribuant à l'augmentation de la capacité d'agir des leaders tout comme des citoyens.

\subsection{Caractéristiques du profil des personnes}

L'analyse du corpus empirique a permis d'identifier cinq caractéristiques du profil des personnes expliquant la participation d'acteurs dans une démarche de développement local en milieu rural :1) leur disponibilité biographique, 2) le fait d'être un(e) élu(e), 3) leur trajectoire d'implication sociale, 4) leur réseau de soutien et 5) le style de leadership. Dans les paragraphes qui suivent, chaque caractéristique est détaillée et expliquée à partir d’appui théorique.

\section{La disponibilité biographique et le fait d'être un(e) élu(e)}

La plupart des participants sont à la retraite dont ils n'ont pas à composer avec des obligations professionnelles et s'ils ont des enfants, sont ceux-ci sont alors adultes (Comeau, 2012). Cependant, ils demeurent très sollicités et occupés, étant donné que la majorité d'entre eux sont des élus municipaux. Selon Vachon (1993), une démarche de développement local s'articule autour des leaders locaux, mais ils ne doivent pas agir seuls. On doit les mettre en relation avec d'autres personnes et instaurer une dynamique de travail d'équipe avec une composition diversifiée de 
membres favorisant le partage des ressources (temps, connaissances, réseaux, etc.).

Un sondage réalisé par Communagir (2015) auprès des élus québécois illustre que ce qui motive les élus à s'impliquer, c'est la possibilité de contribuer à des actions qui auront des impacts positifs sur leur collectivité et qui amélioreront les conditions de vie de la population. Les participants sont conscients qu'ils doivent acquérir des connaissances pour trouver des solutions aux enjeux complexes qui caractérisent les milieux ruraux au Québec. Les apprenants adultes sont motivés à s'impliquer dans des processus d'apprentissage si les connaissances apprises leur permettent de résoudre un problème qui les préoccupe dans l'immédiat (Pouliot, 1997, cité dans Rivard, 2000).

\section{La trajectoire d'implication sociale}

La trajectoire d'implication des participants exerce une influence certaine sur le désir d'engagement social et sur leur présence au sein d'un projet collectif. Les recherches menées par Comeau (2012) ont mis en lumière que les élus étaient déjà très actifs dans leur municipalité, mais pas nécessairement dans des organisations qui avaient des mandats en matière de développement local. Une fois dans leur fonction officielle d'élu, leur implication se module en s'orientant davantage vers ce type d'action. Les résultats de la présente étude vont en ce sens, car les participants étaient engagés dans des organisations appartenant notamment aux secteurs de l'éducation, de la santé et de l'agriculture. Cependant, une fois qu'ils ont été élus, leurs engagements se sont transformés et ils sont davantage impliqués dans des organisations ayant un mandat de développement local dans lesquelles la représentation d’un(e) élu(e) était requise.

Les municipalités sont des organisations qui possèdent différents leviers pour faire du développement. Ces nouvelles perspectives ont indubitablement influencé les élus à développer un intérêt marqué pour le développement local, tout en étant plus aptes à y contribuer. Cela explique en partie pourquoi de multiples expériences de développement local ont prouvé que les groupes de leaders élus jouissent d'une longueur d'avance sur les groupes non élus pour agir dans ce domaine (Vachon, 1993).

\section{Le réseau de soutien}

Le réseautage est un aspect qui a été abordé par les participants et qui s'est avéré un facteur qui les a soutenus dans le développement de leur capacité d'agir. Vachon (1993) mentionne l'importance de la création de réseaux d'échange permettant la circulation de différents types d'informations (connaissances, services et méthodes d'apprentissage), à des échelons différents (local, territorial, régional ou national) et regroupant un maximum de partenaires afin de soutenir la démarche de développement local.

Les participants, qui sont en majorité des élus, jugeaient très important d'être en relation avec différentes personnes ou organisations afin d'avoir accès à de l'information et à du soutien pour les aider à mener à terme leurs différents projets. Comeau (2012) atteste de ce phénomène en expliquant que l'implication des élus s'échelonne souvent sur plusieurs années et, de ce fait, leur permet de développer un réseau de soutien qu'ils peuvent solliciter au besoin. Duperré (2004) ajoute que la capacité de réseautage des élus leur permet de se procurer les ressources nécessaires à la réalisation de leur projet, tout en les confortant dans les décisions à prendre afin que ces dernières soient le plus adaptées au bien commun. Donc, une partie de la capacité d'agir d'une personne désireuse de contribuer à une démarche de développement local réside dans la composition et la diversification des éléments qui constituent son réseau de contacts.

\section{Le style de leadership}

Enfin, l'analyse des résultats fait référence à deux styles de leadership exercés par les élus qui ont eu un effet attractif pour faciliter l'adhésion et la participation des personnes. Tout d'abord, on constate que plusieurs participants ont fait preuve de leadership de compétence. Selon Duperré (2004), cette forme de leadership peut être assumée seulement par les personnes plus âgées qui ont de l'expérience, ce qui se traduit par la connaissance pointue de l'histoire de leur communauté. La totalité des participants correspond à ce profil.

Ensuite, de nombreux participants ont démontré des habiletés politiques qui s'actualisent par des compétences relationnelles pour entrer en relation avec les institutions. Ce type de compétence définit le leadership stratégique (Duperré, 2004), soit le deuxième style de leadership identifié dans cette recherche. 
Les capacités des participants à exercer ces deux types de leadership prennent racine dans leurs attitudes individuelles (écoute, ouverture aux changements et curiosité), dans leurs connaissances acquises au fil du temps et dans leur volonté de travailler en équipe. Le leadership est très contributif au processus de développement local, étant donné que les participants partagent tous la ferme conviction que l'action collective et la concertation au sein de leur communauté sont des composantes incontournables pour arriver à revitaliser leur milieu rural (Duperré, 2004).

\subsection{Participation et compétences comme base à l'apprentissage}

Puisque cette recherche vise à mieux comprendre les phénomènes qui contribuent au développement de la capacité d'agir des personnes impliquées en développement local, il convient d'analyser les données empiriques en fonction de deux composantes du concept d'empowerment individuel : la participation et les compétences.

\section{La participation}

La composante de la participation s'actualise lorsqu'un individu développe son pouvoir d'agir en étant en relation avec d'autres personnes et qu'il participe à une action collective où, ensemble, ils font face à des choix et à des décisions (Ninacs, 2008). L'analyse du profil des répondants nous démontre qu'ils sont impliqués dans leur communauté depuis de nombreuses années et que plusieurs d'entre eux ont occupé des fonctions officielles qui exigeaient de faire des choix et de prendre des décisions pour améliorer le bien commun.

Vachon (1993) affirme qu'il existe quatre formes de participation qui peuvent s'avérer des sources d'apprentissage pour des personnes qui participent à des actions collectives. Les personnes peuvent développer leur capacité d'agir en :

- assistant à une réunion d'information;

- donnant un avis sur une action à mener;

- assumant des responsabilités au sein d'un CA;

- étant partenaire avec une tâche précise au sein d'un projet collectif.

Seulement par leur participation aux rencontres du CA, les participants peuvent exercer la totalité ou plusieurs formes de participation évoquées par
Vachon. Cependant, il faut rester à l'écoute des besoins de chaque membre et offrir différents lieux où chacun se sentira à l'aise de s'exprimer et de participer, comme le mentionne une participante, qui était plus à l'aise de participer dans un comité composé de femmes.

\section{Les compétences}

Les participants sont sollicités par les accompagnateurs du projet à l'étude afin de s'impliquer notamment dans l'animation de rencontres (CA, consultations publiques, atelier dans un colloque, etc.). La trajectoire d'implication communautaire des participants leur a permis de développer des connaissances et des habiletés qui ont facilité leur participation à ce projet novateur.

Selon Ninacs (2008), les connaissances et les capacités permettant la participation et l'exécution de tâches concrètes au sein d'une action collective décrivent la composante des compétences. Les résultats présentés dans les sections sur le profil et les acquis des participants à l'étude démontrent que les répondants possédaient déjà, avant de se joindre à la démarche en 2009, de multiples connaissances et compétences. La majorité des participants ont obtenu un diplôme d'études et ont suivi diverses formations au cours des dernières années. De plus, chacun détenait des connaissances et des habiletés acquises lors d'expérience de travail ou d'implication dans diverses organisations. Ce bagage individuel a nourri la démarche collective qui, elle, en retour, leur a permis de parfaire leurs connaissances.

De ce fait, il existe une relation étroite entre ces deux composantes de l'empowerment. Leur participation au projet a permis aux participants de mieux comprendre les enjeux sociaux, économiques et politiques du territoire, et ces apprentissages ont été possibles grâce aux accompagnateurs et aux relations développées avec différentes personnes et organisations qui ont soutenu le projet.

\subsection{Ingrédients de base pour soutenir le processus d'apprentissage chez les adultes}

Jean et Épenda Muteba Wa (2004) estiment que la maitrise du développement passe nécessairement par des actions de formation afin d'élever le niveau d'éducation de la population et des leaders. Le matériel empirique permet de mettre en lumière quelques 
éléments qui encouragent les personnes à faire des apprentissages, les modes d'apprentissage à privilégier ainsi que les activités facilitant les apprentissages informels.

Selon Brien (1997) et Ninacs (2008), l'appropriation du pouvoir d'agir peut surgir si la personne a quelque chose à perdre et qu'un choix s'impose à elle. L'insatisfaction jugée suffisamment importante dispose à l'action. Il faut dire que ce sentiment d'urgence a été décelé chez les personnes les plus actives des quatre localités, qui représentent en quelque sorte des leaders de leur milieu sur le plan associatif. Le constat d'échec des actions visant la revitalisation de leur milieu au cours des dernières décennies a alimenté leur désir d'innovation sociale. Si le désir d'agir est présent, des méthodes d'enseignement faisant vivre des émotions agréables aux apprenants les motiveront à fournir des efforts pour acquérir les connaissances nécessaires à l'accomplissement d'une tâche, précise Brien (1997).

La variété des méthodes d'enseignement et des stimuli encourage également les adultes à apprendre (Brien, 1997). On sait que les voyages, les réunions, les conférences et la participation aux activités ont sans doute contribué à diversifier les activités dans l'expérience analysée. Cependant, afin de vérifier dans quelle mesure cet aspect a été suffisant, il faudrait le comparer avec d'autres expériences analogues. On comprend qu'il s'agit là d'une limite de la recherche fondée sur un cas unique.

En 1970, les recherches de Tough concluaient que $80 \%$ des apprentissages d'un individu étaient le fruit d'apprentissages informels (Cristol et Muller, 2013). Pour Comeau, Desrosiers et Martin-Caron (2015), il s'agit d'activités qui se produisent au gré du hasard et qui supposent une démarche de planification permettant aux personnes de réaliser une activité. Selon Cristol et Muller (2013), ce mode d'apprentissage dans l'action n'est pas nécessairement reconnu, ni même souvent reconnaissable par les personnes ellesmêmes. Malgré tout, selon ces auteurs, l'intention d'apprendre est une variable clé en matière d'apprentissages informels. Ce désir d'apprendre serait bien présent chez les personnes constituant l'échantillon de cette recherche puisqu'il s'agit de personnes engagées à améliorer les conditions de vie de leurs concitoyens. S’il est possible dans la présente recherche d'identifier des activités informelles d'apprentissage, il est par contre plus difficile de nommer précisément les apprentissages réalisés dans un tel cadre. De même, si les accompagnateurs ont mis les personnes en situation d'apprentissages informels, ils ne l'indiquent pas explicitement dans leur entretien.

Sorohan (1993, cité dans Cristol et Muller, 2013) estime que les apprentissages résultant d'une démarche informelle sont rendus possibles par au moins deux stratégies. La première est d'ordre individuel : la personne est autonome et n'est pas en interaction (Cristol et Muller, 2013). On constate que cette stratégie a été utilisée par certains participants, dont ceux décrits comme des personnes proactives, curieuses et autonomes. Selon quelques indications tirées des entretiens, la lecture des journaux et les recherches sur Internet sont des activités qui relèvent de cette stratégie.

Cristol et Muller (2013) qualifient la seconde stratégie d'apprentissage informel de type relationnel. Elle se concrétise dans les différentes modalités d'appui et d'accompagnement que sont par exemple le mentorat, le coaching, le travail en réseau et le modeling. Cette stratégie a été importante, d'après nos observations, à cause de la prédominance des activités de groupe. À ce sujet, Duperré (2004) précise que les personnes engagées dans une action collective sont nécessairement en relation avec d'autres; ainsi, non seulement cette expérience peut être agréable, mais elle a des retombées positives dans les communautés. Sur le plan des apprentissages, cette stratégie rejoint la perspective de Ninacs (2008), selon laquelle des individus augmentent leur capacité d'agir quand ils sont dans un contexte d'interactions avec d'autres individus.

L'activité d'apprentissage informel la plus souvent mentionnée par les participants est le mentorat. Ce type de mentorat, c'est-à-dire un mentorat pour lequel aucune personne physique n'a été identifiée pour être mentor, est qualifié de diffus par Duperré (2004). On pense ici au réflexe spontané des maires et des accompagnateurs de faire appel, en tout temps, à une personne de leur choix pour les guider dans leur réflexion et leur action.

Brien (1997) attire notre attention sur le modeling: les apprentissages sont faits par imitation. De cette manière, le transfert de savoirs ou de compétences peut s'opérer d'un membre vers un autre membre ou entre les membres et les professionnels. On suppose que le modeling ait pu se faire notamment lors des séances du CA de l'OBNL. De plus, il est possible que les membres aient observé ou écouté un professionnel, puis apprivoisé un vocabulaire ou reproduit une 
façon de faire. Une observation fine du déroulement des activités avec une attention toute particulière à cet aspect doublée d'échanges faits sur place et en situation avec des participants permettraient de documenter davantage ce type d'activité.

Enfin, tout indique que les activités d'apprentissage informel ont des impacts qui vont au-delà du développement de connaissances. À ce propos, les voyages dans d'autres localités, régions ou à l'extérieur du pays ont certes permis aux membres de voir d'autres communautés et de s'interroger sur leur situation en procédant à une analyse comparative. En même temps, ces voyages permettent de se lier à des personnes engagées et déterminées, à s'identifier à elles et à un projet commun. Pour Vachon (1993), il est crucial que les membres du groupe développent une identité collective qui sera la base de leurs actions communes.

\section{Conclusion}

Depuis que le gouvernement libéral est revenu au pouvoir en 2014, il apparaitt que l'époque de l'État accompagnateur du développement est révolue et que nous sommes désormais à l'ère de la «municipalisation» (Chiasson, 2015). Plus que jamais, les communautés rurales doivent prendre en main leur développement. L'actuelle crise sociosanitaire associée à la COVID-19 exacerbe ce besoin d'autonomie. Dans ce contexte, l'approche de développement local est une stratégie intéressante pour trouver des solutions à des problèmes collectifs vécus sur un territoire donné en fonction d'une réalité particulière. Cependant, cette approche nécessite la contribution des acteurs du milieu, et exige qu'ils possèdent des connaissances et des compétences afin de soutenir la réalisation d'actions collectives.

Puisque les enjeux de développement sont nombreux et parfois complexes, les personnes engagées dans des actions collectives auront besoin de parfaire leurs savoirs pour adapter leurs interventions ou leurs projets aux besoins singuliers de leur communauté. En ce sens, cette recherche a permis d'identifier les phénomènes qui contribuent au développement de la capacité d'agir des personnes impliquées dans des démarches de développement local.

Sur le plan méthodologique, un OBNL ayant pour mission d'orchestrer le développement de quatre municipalités dites dévitalisées a été sélectionné (échantillonnage d'un cas unique). Cette organisation présente les caractéristiques d'une démarche de développement local, en plus d'avoir été nommée lauréate par des organisations provinciales sur le plan de l'innovation. Un échantillon significatif d'informateurs a été constitué à partir des administrateurs du CA de l'OBNL (6 des 13 administrateurs) et de professionnels responsables d'offrir de l'accompagnement au groupe.

Cette recherche a mis en lumière divers phénomènes qui contribuent au développement de la capacité d'agir des personnes impliquées en développement local. Certaines dimensions sont liées à des facteurs facilitants du contexte du projet (légitimité, reconnaissance, innovation, mode de fonctionnement et climat du groupe), alors que d'autres sont d'ordre individuel (disponibilité, fonction officielle, trajectoire d'implication sociale, réseau de contacts et style de leadership). De plus, certaines activités en lien avec la participation à diverses activités ou rencontres formelles et informelles se sont démarquées quant à leur façon de favoriser les apprentissages.

La pandémie qui sévit actuellement mobilise de nombreuses ressources et contraint les institutions, les organisations, les territoires, les réseaux locaux d'action collective ainsi que les individus à faire les choses autrement. Depuis mars 2020, des transformations sociales, économiques et politiques s'opèrent. Dans quelques années, des recherches dévoileront les impacts que la pandémie aura eus sur le modèle de développement du Québec. Est-ce que la crise va ébranler les institutions et provoquer une prise de conscience individuelle et collective, au point d'enclencher un changement de paradigme et d'amorcer la construction d'un modèle qui ne reposera plus sur des idéologies néolibérales?

Cette crise représente une opportunité d'innovation afin de revoir nos modes de vie, nos modes de consommation et le développement de nouvelles solidarités pour affronter les défis du XXIe siècle. Sauronsnous saisir cette période transactionnelle pour coconstruire, entre chercheurs, décideurs, fonctionnaires, praticiens et citoyens, une politique d'aménagement et de développement des territoires ruraux du Québec? Le défi est grand, mais pas impossible à relever. Nous avons déjà innové une première fois avec la création de la Politique nationale de la ruralité. Utilisons cette expérience pour nous propulser encore plus loin! 


\section{RÉFÉRENCES}

Ahtik, V. (1989). Développement économique des communautés locales : déplacements dans la réflexion théorique au cours des années quatre-vingt. International Review of Community Development, 22, 141-150. https://doi.org/10.7202/1034025ar

Alberio, M. (2015). Les initiatives locales et les défis des acteurs du milieu face aux coupes et aux changements actuels. Organisations et territoires, 24(3), 53-60. https://doi.org/10.1522/ revueot.v24i3.90

Beaudet, G. et Meloche, J.-P. (2012). L'aménagement du territoire au Québec, le parent pauvre des sciences régionales. Revue d'économie régionale et urbaine, 4, 691-716. https://doi.org/10.3917/reru.124.0691

Bonneau, M.-A. et Dupéré, S. (2020). La participation sociale dans une communauté rurale au Québec : ce qui la facilite, selon les points de vue de personnes engagées. Organisations et Territoires, 29(2), 185-196.

Bourque, D., Comeau, Y., Favreau, L. et Fréchette, L. (dir.). (2007). L’organisation communautaire au Québec. Mise en perspective des principales approches stratégiques d'intervention. Dans L'organisation communautaire au Québec. Fondements, approches et champs de pratique (p. 3-19). Presses de l'Université du Québec.

Bourque, D. et Lachapelle, R. (2010). L'organisation communautaire en CSSS : service public, participation et citoyenneté. Presses de l'Université du Québec.

Brien, R. (1997). Science cognitive et formation. Presses de l’Université du Québec.

Chiasson, G. (2015). Quelle politique régionale en contexte d'austérité ? Organisations et territoires, 24(3), 37-42.

Comeau, Y. (2008). L'approche de développement local en organisation communautaire. Dans D. Bourque, Y. Comeau, L. Favreau et L. Fréchette (dir.), L'organisation communautaire : fondements, approches et champs de pratique (p. 59-79). Presses de l’Université du Québec.

Comeau, Y. (2012). L'engagement social des élus municipaux et leurs représentations du développement local (Cahier $\mathrm{n}^{\circ}$ 1203). Chaire de recherche Marcelle-Mallet sur la culture philanthropique, Université Laval.

Comeau, Y., Desrosiers, M. et Martin-Caron, L. (2015). Intervention collective, mobilisation locale et hébergement des aînés. Presses de l’Université du Québec.

Communagir. (2015). La participation des élus municipaux au développement collectif: résultats du sondage réalisé entre mai et juin 2014 auprès d'élus municipaux québécois. Chantier sur l'évolution des pratiques. https://communagir.org/medias/2016/09/sondage-auxelusvfinale_11_fev._2015.pdf

Côté, L. (2008). Développement des communautés : comment mobiliser et faire participer les citoyens? L'exemple de la communauté de SaintCamille [Mémoire de maitrise, Université de Sherbrooke]. Savoirs UdeS. http://savoirs.usherbrooke.ca/ handle/11143/2575

Cristol, D. et Muller, A. (2013). Les apprentissages informels dans la formation pour adultes. Savoirs, 2(32), 11-59. https://doi.org/10.3917/savo.032.0011

Duperré, M. (2004). L'organisation communautaire : la mobilisation des acteurs collectifs. Presses de l’Université Laval.

Duperré, M. (2008). La rationalité des émotions dans les processus de mobilisation collective. Service social, 54(1), 67-81. https://doi.org/10.7202/018344ar

Fontan, J.-M. (2003). Le développement local, de la contrainte économique au projet politique. Revue Interventions économiques, 30,1-12. https://doi.org/10.4000/interventionseconomiques.993

Glon, É. et Vachon, B. (1996). Enjeux et réalités du développement local au Québec. Revue de géographie de Lyon, 71(3), 261-267. https://doi.org/10.3406/geoca.1996.6230

Hardina, D. (2013). Interpersonal social work skills for community practice. Springer.

Institut de la statistique du Québec. (2020). Évolution de la population totale et de la population dans les ménages privés et les familles de recensement, Québec, 1951-2016. Gouvernement du Québec. https://statistique.quebec.ca/fr/produit/ tableau/evolution-de-la-population-totale-et-de-la-population-dans-les-menages-prives-et-les-familles-de-recensementquebec-1951-2016

Jean, B. (2003). Réussir le développement des communautés rurales : dix conditions gagnantes. Organisations et territoires, 12(2), 19-30. 
Jean, B. (2006). Les études rurales québécoises entre les approches monographiques et typologiques. Recherches sociographiques, 47(3), 503-529. https://doi.org/10.7202/014656ar

Jean, B. (2012). Les territoires ruraux au Québec : vers un modèle de développement territorial durable. Revne d'économie régionale et urbaine, 4, 649-671. https://doi.org/10.3917/reru.124.0649

Jean, B. (2015). La déconnexion régions-métropole au Québec : repenser les politiques publiques pour un développement territorial solidaire. Organisations et territoires, 24(3), 17-22.

Jean, B. et Épenda Muteba Wa, A. (2004). Le capital social et le renforcement des « capacités de développement» des communautés rurales : les enseignements d'une étude canadienne. Revue d'économie régionale et urbaine, 5, 673-694. https://doi.org/10.3917/ reru.045.0673

Joyal, A. (2012). Le développement local au Québec : trois décennies de contributions. Revue d'économie régionale et urbaine, 4, 673-690. https://doi.org/10.3917/reru.124.0673

Joyal, A. (2015). Quel avenir pour les régions périphériques? Organisations et territoires, 24(3), 23-28. https://doi.org/10.1522/ revueot.v24i3.86

Joyal, A., Pouquay, F. et El-Batal, K. (2005). La politique québécoise de la ruralité : le développement local à l'épreuve. Géographie, économie, société, 7(2), 193-211. https://doi.org/10.3166/ges.7.193-211

Lachapelle, R. et Bourque, D. (2020). Des collectivités résilientes en contexte politique difficile : la contribution de l'intervention collective. Organisations et territoires, 29(2), 117-126. https://doi.org/10.1522/revueot.v29n2.1155

Ladouceur, S. (2018). Bulletin d'analyse : indice de vitalité des territoires. Institut de la statistique du Québec. https://statistique. quebec.ca/fr/fichier/bulletin-danalyse-indice-de-vitalite-economique-des-territoires-edition-2018.pdf

Lavoie, J. et Panet-Raymond, J. (dir.). (2020). La pratique de l'action communautaire (4éd.). Presses de l’Université du Québec.

Ministère des Affaires municipales et de l'Habitation (MAMH). (2020). L'organisation municipale au Québec en 2020. Gouvernement du Québec. https://www.mamh.gouv.qc.ca/fileadmin/publications/organisation_municipale/ organisation_territoriale/BRO_OrganisationMunicipale_2020.pdf

Morin, L. (2016). Compétences et pouvoir d'agir : étude sur les apprentissages réalisés par des acteurs impliqués dans une expérience de développement local [Mémoire de maitrise, Université Laval]. Corpus ${ }^{\mathrm{UL}}$. https:// corpus.ulaval.ca/jspui/ bitstream/20.500.11794/27396/1/33082.pdf

Ninacs, W. A. (2008). Empowerment et intervention: développement de la capacité d'agir et de la solidarité. Presses de l'Université Laval.

Parent, F. (2011). Du matériel au symbolique : fondements et limites des études rurales québécoises contemporaines. Recherches sociographiques, 52(2), 335-351. https://doi.org/10.7202/1005665ar

Richard, B. (2000). Psychologie des groupes restreints. Presses Inter Universitaires.

Rivard, P. (2000). La gestion de la formation en entreprise : pour préserver et accroître le capital compétence de votre organisation. Presses de l’Université du Québec.

Sabourin, P. (2010). L'analyse de contenu. Dans B. Gauthier (dir.), Recherche sociale: de la problématique à la collecte des données (5e éd., p. 415-444). Presses de l’Université du Québec.

Savoie-Zajc, L. (2010). L'entrevue semi-dirigée. Dans B. Gauthier (dir.), Recherche sociale : de la problématique à la collecte des données (5e éd., p. 337-360). Presses de l’Université du Québec.

Simard, J.-F. et Leclerc, Y. (2008). Les Centres locaux de développement 1998-2008 - Une gouvernance en mutation : entre participation citoyenne et imputabilité municipale. Revue canadienne des sciences régionales, 31(3), 615-636. https://idjs.ca/ wp-content/uploads/v31n3-simard-leclerc.pdf

Solidarité rurale du Québec (SRQ). (2018). Les milieux ruraux du Québec : portraits régionaux. Coop Carbone. https://tousruraux.quebec/wp-content/uploads/2018/05/portrait-milieuxruraux-coopcarbone-2018.pdf

Stake, R. E. (2005). Qualitative case studies. Dans N. K. Denzin et Y. S. Lincoln (dir.), The SAGE bandbook of qualitative research (3e éd., p. 443-466). SAGE. 
Statistique Canada. (2021). Recensement de 2016. https://www12.statcan.gc.ca/census-recensement/2016/dp-pd/prof/ index.cfm? Lang $=\mathrm{F}$

Torre, A. (2015). Théorie du développement territorial. Géographie, économie, société, 17(3), 273-288. https://doi.org/10.3166/ges.17.273-288

Vachon, B. (1993). Le développement local : théorie et pratique, réintroduire l'bumain dans la logique de développement. Gaëtan Morin Éditeur. 